\title{
Characteristics of Flare-Productive Sunspot Groups
}

\author{
Takako T. Ishii \\ Kwasan and Hida Observatories, Kyoto University, Yamashina-ku, \\ Kyoto, JAPAN, 607-8471 \\ Hiroki Kurokawa \\ Kwasan and Hida Observatories, Kyoto University, Yamashina-ku, \\ Kyoto, JAPAN, 607-8471 \\ Tsutomu T. Takeuchi \\ Division of Particle and Astrophysical Sciences, Nagoya University, \\ Nagoya, JAPAN 464-8602
}

\begin{abstract}
The mechanism of flare energy build-up is one of the most fundamental questions in the solar flare study, but is still to be solved. From the review of the previous studies, we notice that the formation process of the magnetic shear in an active region should be essential for the flare energy build-up mechanism. Based on this idea, we make detailed studies of the active region evolutions using high resolution $\mathrm{H} \alpha$ images obtained with the $60 \mathrm{~cm}$ Domeless Solar Telescope at Hida Observatory, Kyoto University.

We study sunspot proper motions and evolutionary changes of $\mathrm{H} \alpha$ fine structures and magnetic fields in active regions NOAA 5395 (Ishii et al. 1998) and NOAA 4201 (Ishii et al. 2000). To explain the evolutionary characteristics found from the analysis of these two active regions, we propose schematic models of twisted flux bundles emerging from the convection zone. We also found that the occurrence of high flare activity in each active region was restricted to the rapidly emerging region of the twisted flux bundle. In conclusion, we suggest that the emergence of the twisted flux bundle should be the key to high flare-productivity of the sunspot group, or the flare energy build-up mechanism.
\end{abstract}

Acknowledgments. We made extensive use of National Solar Observatory Digital Library and the Space Physics Interactive Data Resource (SPIDR).

\section{References}

Ishii, T. T., Kurokawa, H., \& Takeuchi T. T. 1998, ApJ, 499, 898

Ishii, T. T., Kurokawa, H., \& Takeuchi T. T. 2000, PASJ, 52, 337 